\title{
Lumen
}

Selected Proceedings from the Canadian Society for Eighteenth-Century Studies

\section{Le théâtre de Marivaux et la Terreur}

\section{Martin Nadeau}

Volume 22, 2003

URI : https://id.erudit.org/iderudit/1012256ar

DOI : https://doi.org/10.7202/1012256ar

Aller au sommaire du numéro

Éditeur(s)

Canadian Society for Eighteenth-Century Studies / Société canadienne d'étude du dix-huitième siècle

ISSN

1209-3696 (imprimé)

1927-8284 (numérique)

Découvrir la revue

Citer cet article

Nadeau, M. (2003). Le théâtre de Marivaux et la Terreur. Lumen, 22, 15-26.

https://doi.org/10.7202/1012256ar

Copyright $@$ Canadian Society for Eighteenth-Century Studies / Société canadienne d'étude du dix-huitième siècle, 2003
Ce document est protégé par la loi sur le droit d'auteur. L'utilisation des services d'Érudit (y compris la reproduction) est assujettie à sa politique d'utilisation que vous pouvez consulter en ligne.

https://apropos.erudit.org/fr/usagers/politique-dutilisation/ 


\section{Le théâtre de Marivaux et la Terreur}

Car outre les choses dessus dites, la nature des peuples est changeante, et il est aisé de les persuader d'une chose, mais difficile de les garder dans cette persuasion. Aussi faut-il y donner si bon ordre que lorsqu'ils ne croiront plus, on leur puisse faire croire par force.

Machiavel, le Prince, VI.

La renaissance du Théâtre-Italien à Paris au début du XVIII ${ }^{\mathrm{e}}$ siècle s'inscrit dans le contexte de la mort de Louis XIV en 1715, et la première tâche pour Philippe d'Orléans est bien de se distinguer de son prédécesseur. Louis XIV, rappelons-le, a congédié pour son irrévérence l'ancienne troupe du Théâtre-Italien en 1697. Mme de Maintenon, passée subrepticement à la dévotion, aurait voulu empêcher la représentation de la Finta Matrigna (la Fausse Prude, une comédie attribuée tantôt à Fatouville, tantôt à Lenoble) dans laquelle elle s'est vue raillée; à tout le moins, elle a craint les applications que le public pouvait faire ${ }^{1}$. Le Régent demande au duc de Parme de lui envoyer une nouvelle troupe de comédiens italiens avec pour capo comico Luigi Riccoboni ${ }^{2}$. Le nouvel Arlequin, Tommaso Visentini, dit Thomassin, Pierre-François Biancolelli (le fils de l'ancien Arlequin) dans le rôle du troisième zanni Pedrolino ou Pierrot, non moins que l'innamorato Lélio incarné par Riccoboni lui-même, ont assuré le succès de cette nouvelle troupe jouant des pièces du répertoire de l'ancienne, comme l'Heureuse Surprise présentée devant une salle comble le 18 mai 1716 au Palais-Royal ${ }^{3}$.

1 Voir à ce sujet François Moureau, De Gherardi à Watteau. Présence d'Arlequin sous Louis XIV, Paris, Klincksieck, 1992.

2 Voir Guy Boquet, «La Comédie Italienne sous la Régence. Arlequin poli par Paris», Revue d'histoire moderne et contemporaine, 24, 2, avril-juin 1977, p. 189-214.

3 Voir Thomas-Simon Gueullette, Notes et souvenirs sur le Théâtre-Italien au XVIII siècle, publiés par J.-E. Gueullette, Paris, 1938; Genève, Slatkine reprints, 1976, p. 26-27. 
$C^{\prime}$ est en cherchant à diversifier encore davantage son répertoire, et surtout à réconcilier le milieu des comédiens avec celui des lettrés, que Riccoboni fait entrer Marivaux en scène. Avec des pièces comme Arlequin poli par l'amour (1720), la Surprise de l'amour (1722) ou la Double Inconstance (1723), ce dernier a contribué significativement à la fortune de ce théâtre dans la première moitié du XVIII ${ }^{\mathrm{e}}$ siècle ${ }^{4}$. Si par la suite Marivaux peut être considéré davantage comme un auteur de la Comédie-Française, ses "classiques", le Jeu de l'amour et du hasard (1730), la Mère confidente (1735), les Fausses Confidences (1736) et l'Épreuve (1740), ont souvent occupé avec succès la scène du Théâtre-Italien dans la seconde moitié du siècle, incluant la période révolutionnaire, et ce quoique des historiens aient parlé d'une «œuvre peu estimée au XVIII ${ }^{\mathrm{e}}$ siècle $^{5}$ ».

Du vivant de Marivaux (1688-1763), la critique ne fonctionne guère «à chaud», en raison notamment de l'absence de presse quotidienne. Les critiques littéraires sont écrites en marge des représentations et parfois elles ne se fondent que sur la pièce imprimée. Elles ont peu d'effet sur les spectateurs, qui jugent, eux, dans l'instant et dans la chaleur de la création ${ }^{6}$. Pour la période qui a fait l'objet de ma recherche, 1770-1799 ${ }^{7}$, la naissance du premier quotidien parisien, le Journal de Paris, en 1777, et l'essor de la presse dans les années subséquentes, avec l'apparition d'un deuxième quotidien dans la capitale, la Chronique de Paris, en 1789, et de journaux spécialisés comme le Journal des spectacles en 1793, enrichissent considérablement les ressources de l'historien. Les comptes rendus que renferment ces quotidiens permettent de reconstituer en partie les moments éphémères que sont les spectacles de théâtre, en considérant tour à tour le contexte sociopolitique, les rôles des comédiens et du public, aux côtés de celui de l'auteur, dans la configuration du sens d'une pièce. Mais, d'abord, ils témoignent des pièces que le public de l'époque a accueillies avec le plus d'enthousiasme, de «transport», dont celles de Marivaux, en particulier pendant la Terreur.

S'attachant à l'ensemble du répertoire des théâtres parisiens durant la décennie révolutionnaire, le recueil de statistiques réalisé sous la direc-

4 Voir Xavier de Courville, Lélio premier historien de la Comédie Italienne et premier animateur du théâtre de Marivaux, Paris, Librairie théâtrale, 1958.

5 Marivaux, Théâtre complet, Paris, Seuil, 1964, préface de Jacques Scherer, p. 7.

6 Voir Henri Lagrave, Marivaux et sa fortune littéraire, Bordeaux, Ducros, 1970, p. 36.

7 Voir Martin Nadeau, "Théâtre et esprit public: le rôle du Théâtre-Italien dans la culture politique parisienne à l'ère des Révolutions (1770-1799)», Montréal, Université McGill, thèse de doctorat, 2001. 
tion d'Emmet Kennedy établit que Marivaux, dans le palmarès des cent auteurs les plus joués, se situe au $33^{\mathrm{e}}$ rang, avec un total de 559 représentations entre 1789 et $1799^{8}$. Ses pièces, comme le Jeu de l'amour et du hasard donnée à elle seule à 169 reprises au cours de cette période ${ }^{9}$, ont été représentées deux fois plus souvent que celles de l'auteur dramatique le plus spontanément cité dans les études sur le théâtre révolutionnaire, soit Marie-Joseph Chénier, au $69^{\mathrm{e}}$ rang de ce palmarès, avec 283 représentations au total. Il faut rappeler à cet égard l'importance de la loi sur la liberté des théâtres votée le 13 janvier 1791, qui a porté la «théâtromanie» du XVIII ${ }^{\mathrm{e}}$ siècle à son paroxysme en autorisant la prolifération des salles de spectacles dans la capitale. Cette loi a entre autres permis à un public très varié, composé de spectateurs plus lettrés au Théâtre de la citoyenne Montensier et au Théâtre de la République, plus «populaires» au Théâtre des sans-culottes, au Théâtre patriotique ou au Théâtre des délassements comiques, de découvrir ou de redécouvrir Marivaux ${ }^{10}$.

La pièce les Fausses Confidences est entrée dans le répertoire du Théâtre de la Nation — ci-devant Comédie-Française — le 15 juin 1793, à la suite d'une représentation qui l'a consacrée durablement parmi les meilleures de Marivaux; le Journal de Paris souligne que «le public s'y est porté en foule ${ }^{11} »$. Le 22 juillet 1793, en pleine effervescence politique conduisant à la proclamation de la Terreur, la Chronique de Paris, à travers un compte rendu de l'Épreuve, élabore sur l'esprit et l'«usage du monde» qui sont requis pour bien jouer Marivaux ${ }^{12}$. Ce même quotidien avait remarqué,

8 Voir Emmet Kennedy, Marie-Laurence Netter, John P. McGregor et Mark V. Olsen, Theatre, Opera and Audiences in Revolutionary Paris. Analysis and Repertory, Westport, Greenwood Press, 1996, p. 383, tableau 5, «Top 100 Authors by Number of Performances, 1789-1799".

9 Voir ibid., p. 382, tableau 4, «Top 50 Plays by Number of Performances, 1789-1799». Le Jeu de l'amour et du hasard figure au $42^{\mathrm{e}}$ rang.

10 Ces cinq salles correspondent aux lieux de représentation du Jeu de l'amour et du hasard entre 1789 et 1799 . Pour la description de la trentaine de théâtres que compte Paris pendant la décennie révolutionnaire, voir Guiseppe Radicchio et Michèle Sajous d'Oria, les Théâtres de Paris pendant la Révolution, traduction de Laura Casati et Françoise Lenoir, Paris, Bibliothèque de la ville de Paris, 1990.

11 Cité par Jacques Scherer, Marivaux. Théâtre complet, op. cit., notice sur les Fausses Confidences, p. 446.

12 Voir la Chronique de Paris, 203, 22 juillet 1793, p. 2. La chute des Girondins est du 2 juin, alors que l'insurrection vendéenne est à son paroxysme (prise de Saumur le 9 juin; prise d'Angers, le 18); Marat sera assassiné le 13 juillet; Robespierre entrera au Comité de salut public le 27 du même mois; la surveillance des spectacles sera décrétée le 2 août 1793 . 
au début de juillet 1793, que plus "[ $\left[1^{\prime}\right]$ horizon politique se rembrunit, plus l'esprit a besoin de trouver au théâtre une illusion de plaisir que sa situation intérieure ne peut lui procurer", d'où, selon lui, le succès rencontré par des pièces où règne une "gaîté fine», en contraste avec la brutale réalité de l'été 1793: «\& l'on peut assurer que la réalité des scènes noires nous a dégoûté de leur représentation théâtrale ${ }^{13}$ ».

Comme le suggèrent ces journaux, il faut d'abord expliquer le succès des intrigues de Marivaux par le fait qu'elles offraient, à un moment que par euphémisme nous pouvons qualifier de triste, un divertissement gai ou un exutoire. Une seconde explication réside dans la nature profonde de son théâtre. La critique sociale et la satire des mœurs - en conformité avec la devise du Théâtre-Italien, Castigat ridendo mores - sont omniprésentes au sein de ces intrigues amoureuses où l'on pratique l'autopsie des passions humaines, mais ces dernières ne concernent pas uniquement l'amour. Dans la tradition du théâtre à l'italienne, il s'agit aussi de la vie politique entendue comme dynamique d'un corps social aux prises avec ses tensions, ses crises, ses conflits de sexes, de générations et de rangs sociaux. A l'Arlequin sauvage de Delisle de la Drevetière, qui met en scène en 1721, bien avant Rousseau, un Arlequin-Huron dénonçant la corruption de la civilisation et célébrant les vertus de l'état de nature, fait écho l'Ile des esclaves de Marivaux (1725), où des maîtres naufragés doivent échanger leur rôle social avec leurs valets, moins dans une perspective «jacobine» que dans l'esprit d'une autocritique et d'une réconciliation sociale fondée sur la raison. Dans un contexte politique où les passions et les ressorts de la séduction, de la manipulation et de la corruption font éprouver leur dynamique avec acuité, l'univers de l'auteur des Fausses Confidences a pu faire entendre de nouvelles résonances.

Une comédie de Marivaux opère d'abord par le jeu des acteurs et des actrices, plus que par les intrigues, une dialectique du langage et des mots qui servent indifféremment à mentir ou à dire la vérité. Son théâtre, où les acteurs cachent ce qu'ils pensent et disent ce qu'ils ne pensent pas, doit être considéré non seulement dans sa dimension textuelle, mais aussi à travers le jeu des comédiens en interaction avec le public ${ }^{14}$.

La présence d'une actrice comme Gianetta Benozzi, dite Sylvia, la «muse» de Marivaux, dont on dit qu'elle a pu jouer jusqu'à l'âge de

14 Voir d'Henri Lagrave, qui s'est intéressé beaucoup à cette question du public, outre Marivaux et sa fortune littéraire, op. cit., p. 52-53, le Théâtre et le public à Paris de 1715 à 1750, Paris, Librairie C. Klincksieck, 1972. 
cinquante ans les jeunes innamorata d'une manière crédible, a compté pour beaucoup dans les premiers succès qu'a rencontrés l'auteur ${ }^{15}$. Dans la seconde moitié du XVIII ${ }^{e}$ siècle, Mlle Contat, qui a notamment incarné Araminte dans les Fausses Confidences en 1793, a su réunir des éloges comparables à ceux accordés à Sylvia. Les Comédiens-Français ont choisi cette pièce pour leur rentrée au lendemain de la Terreur, le 16 août 1794, et le succès fut tel que le spectacle ne dura pas moins de huit heures: "Mais où tous les suffrages ont éclaté avec la plus bruyante expression, c'est dans les Fausses Confidences; la citoyenne Contat, chargée du rôle d'Araminte, a étonné et enlevé tous les spectateurs; ce jeu magique, qui n'appartient qu'à elle, a électrisé tout le monde ${ }^{16}{ }^{\prime}$.

Le succès des pièces de Marivaux dans le contexte révolutionnaire, comme le Dénouement imprévu en 1789, peut être attribuable aussi aux applications qu'en faisait le public, et plus particulièrement cette catégorie de spectateurs que l'on nomme sous l'Ancien Régime «les plaisants du parterre» et sous la Révolution «les patriotes du parterre». Ces plaisants ou ces patriotes du parterre interviennent activement dans la représentation du spectacle pour exprimer, verbalement ou non, c'est-à-dire par des sifflets, des applaudissements, des bruits, parfois de violents éternuements, leurs opinions. Certaines répliques du Dénouement imprévu ont été investies d'accents nouveaux par le public patriote du Théâtre de la Nation, qui appliquait la passion de l'égalité animant Paris en cet été 1789 à la représentation du spectacle. Il en va ainsi avec cette réplique que prononce Pierrot, un paysan au patois coloré (comme quoi c'est bien l'ensemble du corps politique qui est donné à voir sur les scènes de théâtre au XVIII ${ }^{\mathrm{e}}$ siècle):

D'un sang noble? (s'écrie-t-il après avoir entendu son maître déplorer le fait que son rival soit un gentilhomme,) Queu guiable d'invention d'avoir fait comme ça du sang de deux façons, pendant qu'il viant du même ruisseau?

15 Voir la notice sur Sylvia, de son vrai nom Gianetta ou Jeanne-Rose-Guyonne Benozzi (née à Toulouse vers 1701, morte à Paris en 1758), dans Émile Campardon, les Comédiens du Roi de la troupe italienne pendant les deux derniers siècles, Paris, 1880; Genève, Slatkine reprints, 1970, p. 12-19.

16 Alphonse Aulard, Paris pendant la réaction thermidorienne et sous le Directoire, Paris, Le Cerf, 1898-1902, 5 volumes; voir vol. 1, «Messager du soir du $1^{\text {er }}$ fructidor», p. 48. Le jeu des autres comédiens lors de cette représentation a été aussi apprécié: «le citoyen Fleury, par la décence et l'abandon fougueux de son amour, et la citoyenne Devienne, par sa gaîté piquante, ont ajouté au charme de ce spectacle vraiment ravissant». 
La Chronique de Paris, en parlant de l'acteur qui a incarné ce Pierrot au tout début de la Révolution, rapporte que «M. Belmont a mis dans son jeu autant de naturel qu'il y en a peu dans son rôle, dont les allusions, vivement saisies \& applaudies, ont seules soutenu la pièce ${ }^{17}{ }$.

Cette question des allusions ou applications est cruciale dans l'appréhension de la vie théâtrale au XVIII ${ }^{\mathrm{e}}$ siècle et en particulier pendant les moments de crise politique comme la Révolution. Les comptes rendus des spectacles, ou encore les rapports des agents du gouvernement chargés de les surveiller, recèlent de nombreuses considérations à cet égard et témoignent de l'importance du rôle joué par le public dans le succès ou la chute d'une pièce, en dépit parfois de l'auteur et des acteurs. Par ailleurs, ce public est ciblé selon chaque théâtre; nous savons par exemple qu'au Théâtre Feydeau la salle était fréquentée assidûment par la «jeunesse dorée». Lors d'une représentation des Fausses Confidences donnée devant cette salle en octobre 1796, dans un contexte de ressentiment généralisé envers la Terreur et la violence révolutionnaire, le püblic a applaudi à trois reprises la réplique «Les gens de bien sont sans fortune, et elle a passé aux gens de rien», moins parce qu'il appréciait la versification de l'auteur, le jeu ou le ton de l'acteur, que parce qu'elle prêtait à des applications au contexte politique immédiat ${ }^{18}$.

Par le phénomène des applications, le politique surgit parfois là où on l'attend le moins. D'ailleurs, il faut remarquer que ce ne sont pas les pièces les plus "politiques» de Marivaux qui sont jouées avec succès durant cette période, comme par exemple l'île des esclaves où, au sein de la tradition des rituels d'inversion dans laquelle la commedia dell'arte s'inscrit, des esclaves deviennent les maîtres et les maitres des esclaves dans le cadre d'une réconciliation sociale qui cherche moins la subversion de l'ordre politique que sa reproduction à travers son inversion, le temps d'une pièce ou d'un carnaval. Ce fait est noté en juillet 1793 par le critique de la Chronique de Paris qui suggère la représentation de nouvelles pièces de Marivaux, comme le Prince travesti, ou encore la mise en scène de ses

17 La Chronique de Paris, 38, 30 septembre 1789, p. 151.

18 Voir Alphonse Aulard, op. cit., «Rapport du bureau central du 23 vendémiaire» (14 octobre 1796), «Spectacles», p. 517: «Les spectacles ont été assez tranquilles. Les allusions toujours saisies et courues au théâtre de Feydeau, où l'on donnait les Fausses Confidences; on a applaudi à trois reprises cette phrase: "Les gens de bien sont sans fortune, et elle a passé aux gens de rien"». De même, au cours d'une représentation de la Surprise de l'Amour, toujours au Théâtre Feydeau en octobre 1796, la réplique «En vérité il n'y a que les honnêtes gens de tourmentés» a été «beaucoup applaudi[e]». Voir le rapport du bureau central du 30 octobre 1796, p. 548. 
romans, comme le Paysan parvenu, dans lesquels la question de la hiérarchie sociale nourrit une intrigue constituée, dans ce contexte orageux, de significatives allusions politiques ${ }^{19}$. La direction du Théâtre Montensier, en proposant l'Ile des esclaves sous le titre du Naufrage d'Arlequin le 27 octobre 1793, avait estimé également "qu'un pareil ouvrage étoit parfaitement à l'ordre du jour». Le public toutefois en a jugé autrement, en interrompant cette représentation. Lorsque le «seigneur» Arlequin, après avoir vainement ordonné à son ancien maître Iphicrate d'approcher des sièges, s'est écrié «La République le veut», une application à la volonté omnipotente de la République française en cette période de Terreur a été faite. Le Journal des spectacles relate qu'alors «les ennemis de la pièce crièrent plus fort, et prétendirent qu'elle n'avoit été faite que pour tourner la république en ridicule ${ }^{20} \gg$. Ce n'est pas l'acteur ou la direction du Théâtre Montensier qui est en cause, comme l'a cru le public en pensant que le mot «république» avait été ajouté à dessein, mais tout simplement le fait que Marivaux, lors de la création de cette pièce en 1725 , avait lui-même employé ce terme, dans un contexte bien différent naturellement de celui de la république jacobine.

Ces considérations sur l'importance du rôle du public au théâtre ne doivent pas pour autant mener à minimiser l'importance d'un auteur dont c'est précisément la qualité de lettré qui a attiré l'attention de Luigi Riccoboni. On peut parler à cet égard de l'énigme du "marivaudage», un terme employé par de nombreuses personnes qui n'ont jamais lu Marivaux et ne se soucient ni de lui ni de son œuvre, comme le remarque Jacques Scherer ${ }^{21}$. Marivaux partage ainsi avec Machiavel et Sade l'insigne honneur pour un homme de lettres d'ajouter, malgré lui et uniquement par la résonance de son œuvre, un substantif d'usage courant à la langue française.

Le théâtre de Marivaux agit comme un mécanisme de dévoilement des masques que se donnent les comédiens, d'abord à travers les déguisements successifs dont ils s'affublent pour sonder les cœurs de leurs soupirants. Il s'agit donc de masques corporels, mais aussi des masques du langage, de l'esprit, ou des discours à travers lesquels ils expriment les ambiguïtés de leurs sentiments et de leurs passions. Enfin, il s'agit aussi des masques sociaux qui composent le corps politique. Le temps d'un «rendez-vous de peu de durée», dans le Jeu de l'amour et du

19 Voir la Chronique de Paris, 203, 22 juillet 1793, p. 2.

20 Le Journal des spectacles, 119, 27 octobre 1793, p. 940-942.

21 Jacques Scherer, préface au Théâtre complet de Marivaux, op. cit., p. 7. 
hasard, l'innamorata Sylvia prend les habits de sa servante Lisette pour sonder le cœur de Dorante qui, de même, à l'insu des précédentes mais pas du public complice, s'affublera des vêtements de son serviteur Arlequin afin d'espionner son amoureuse et de mener une reconnaissance sur un terrain inconnu. Le personnage du valet Dubois dans les Fausses Confidences constitue peut-être l'archétype du conspirateur marivaudien: «Mais voici l'heure à peu près qu'il vous a dit qu'il arriverait. (Il cherche et regarde.) N'y a-t-il là personne qui nous voie ensemble? Il est essentiel que les domestiques ici ne sachent pas que je vous connaisse» (I, 2); ou encore dans la scène 16 du même acte: «Partez; aussi ai-je un petit avis à donner à Marton. Il est bon de jeter dans tous les esprits les soupçons dont nous avons besoin». Dans une période de soupçon et de méfiance exacerbée comme celle de la Terreur, à travers la quête d'une transparence du langage et des comportements, la chasse aux hypocrites, la crainte de l'intrigue, la volonté forcenée d'abattre les masques, eux qui caractérisent le fanatisme de la vertu ${ }^{22}$, les pièces de Marivaux peuvent rendre compte d'une résistance à la représentation du corps politique définie par les autorités révolutionnaires de l'an II.

La Feuille de salut public a exprimé à maintes reprises son indignation envers ces «spectateurs qui applaudissent par habitude à ces gentillesses de l'ancien régime»; «ll n'est plus de valets, parce qu'il n'y a plus de maîtres», confie cet organe du Comité de salut public ${ }^{23}$. Pendant la Terreur, outre le triomphe de «l'Incorruptible» Robespierre, on assiste aussi à celui de l'ambition d'une régénération intégrale de la nature humaine dans laquelle les passions égoïstes de chaque individu, de chaque faction, seraient éradiquées au profit de la fondation d'un corps politique égalitaire, homogène et transparent.

À l'instar de la question des masques, corporels, langagiers ou sociaux, celle de la corruption est tout aussi prégnante dans le contexte révolutionnaire. Elle anime la plupart des intrigues amoureuses de Marivaux: l'intérêt personnel ou particulier d'un personnage d'autorité, familiale ou sociale, compromet la réalisation des désirs des amoureux qui doivent, à force de ruse, de raison et d'audace, contourner les obstacles sur leur passage. Pour reprendre encore une fois l'exemple des Fausses Confidences, le valet Dubois de l'amoureux Dorante, anticipant à

22 Hannah Arendt, dans Essai sur la Révolution (traduction de Michel Chrestien, Paris, Gallimard, coll. «Tel», 1967, p. 138), remarque: «la Révolution, avant de dévorer ses enfants, les avait démasqués».

23 Le Journal des spectacles, 141, 20 novembre 1793, p. 1119-1120: «Réflexions sur le théâtre, extraites de la Feuille de salut public». 
la fois Figaro, pour qui la noblesse réside non dans la naissance mais dans le caractère, et certains personnages des Liaisons dangereuses, "fera jouer toutes ses batteries» (I, 17) afin de faire parler et triompher l'amour de Dorante pour Araminte. Il conduit avec courage et virtuosité l'amoureux vers sa cible:

Oh! vous m'impatientez avec vos terreurs. [...] Nous sommes convenus de toutes nos actions, toutes nos mesures sont prises; je connaîs l'humeur de ma maîtresse, je sais votre mérite, je sais mes talents, je vous conduis; et on vous aimera, toute raisonnable qu'on est; on vous épousera, toute fière qu'on est; et on vous enrichira, tout ruiné que vous êtes, entendez-vous? Fierté, raison et richesse, il faudra que tout se rende. Quand l'amour parle, il est le maître; et il parlera [...] $(\mathrm{I}, 2)$.

Mme Argante, le premier obstacle, souhaite que sa fille Araminte épouse le comte de Dorimont et qu'elle choisisse pour intendant le protégé de ce dernier et non Dorante, afin de terminer un procès qui les divise depuis longtemps. A l'acte II intervient le comte de Dorimont qui tentera, à l'instar de Mme Argante, de corrompre l'intendant Dorante: «Parlons à cet intendant; s'il ne faut que de l'argent pour le mettre dans nos intérêts, je ne l'épargnerai pas» (II, 4), en vain. Marton, suivante d'Araminte et complice de Dubois, met en garde le comte: «Oh! non! ce n'est point un homme à mener par là, c'est le garçon de France le plus désintéressé»; «Tant pis; (dit le comte,) ces gens-là ne sont bons à rien" (II, 11). Araminte ne partagera pas cet avis; au rythme des intrigues des valets qui alternativement retardent (avec Arlequin) et accélèrent (avec Dubois) l'action, elle sera conquise. Devant la confidence faite par Dorante des machinations et manipulations - des fausses confidences du conspirateur Dubois, elle conclut la pièce en prononçant ces mots d'une résonance que l'on pourrait qualifier de machiavélienne: «il est permis à un amant de chercher les moyens de plaire, et on doit lui pardonner, lorsqu'il a réussi» (III, 12).

Cette résonance machiavélienne dans l'œuvre de Marivaux n'est pas étrangère aux raisons qui expliquent le succès de son théâtre au cours d'une période de crise politique comme la Terreur. À travers les crises politiques qui déchirent la péninsule italienne à l'époque de la Renaissance, des humanistes comme l'Arioste (1474-1533), l'Arétin (1492-1556) ou Machiavel (1469-1527) écrivent des comédies amoureuses d'inspiration classique qui sont dites "érudites», pour les distinguer des pièces improvisées, car elles reposent au contraire de celles-ci sur un texte fixe. D'aucuns considèrent la Mandragore de Machiavel, composée vers 1520, comme le chef-d'œuvre du genre. Franco Tonelli a dressé un parallèle très intéressant entre cette intrigue amoureuse et l'œuvre politique de Machiavel, en particulier le Prince, où le Florentin exprime une vision du 
monde en termes de combat incessant entre l'humanité et une fortune capricieuse $^{24}$.

Le succès politique du prince dépend de deux facteurs qui sont des avatars de la virtù: premièrement, un pouvoir raffiné de persuasion, une rhétorique et, deuxièmement, un courage viril, une virtuosité afin de se rendre complice la fortune. La fin et le sens ultime de la sexualité, comme métaphore du politique, dépendent de la capacité du prince ou de l'amoureux à séduire la fortune ou l'amoureuse. Des idées clés de l'œuvre politique de Machiavel, comme par exemple la moralité à "géométrie variable», c'est-à-dire l'idée que la morale est variable en fonction de circonstances imprévisibles, se trouvent au cœur d'une pièce comme la Mandragore. A la fin de la pièce, le protagoniste Callimaco (amoureux-prince), qui a cherché à séduire Lucrezia (amoureuse-fortune), a pleinement réussi en rhétorique (grâce à l'intervention d'un confesseur complice, le frère Timoteo), en apparence (par l'artifice de la mandragore) et en fait: Lucrezia est convaincue que ce qui lui est arrivé a été voulu par le ciel et elle se réjouit de son destin. «Sage et pieuse, c'est par obéissance à sa mère, à son confesseur et à son mari, qu'elle prend un amant et découvre l'amour», pour reprendre la formule de Madeleine Lazard $^{25}$. Le secrétaire florentin indique ainsi qu'il n'y a pas de permanence hiérarchique entre le dominant et le dominé, puisque le thème essentiel et le plus profond de la sexualité des corps humains comme métaphore des corps politiques réside dans l'ambiguité de la lecture des signes. Ces signes n'ont pas de signification transcendantale, leur signification est pragmatique et toujours ouverte aux contingences des interprétations futures. La sexualité et le pouvoir politique exigent la maîtrise de leur inéluctable théâtralité.

C'est dans un récit dialogué intitulé le Chemin de la Fortune ou le Saut $d u$ fossé, qui a été publié dans sa feuille le Cabinet du philosophe en 1734, que Marivaux exploite le plus systématiquement le paradigme machiavélien. Ce récit dialogué est peut-être plus qu'un simple "délassement intellectuel évoquant la question des conditions de la réussite sociale ${ }^{26}{ }_{\gg}$. Au moment où il a été composé, ce récit a pour cadre l'actualité

24 Voir Franco Tonelli, «Machiavelli's Mandragola and the Signs of Power», dans Drama, Sex and Politics, Cambridge, Cambridge University Press, coll. «Themes in Drama», 1985, p. 35-54.

25 Madeleine Lazard, la Comédic humaniste au XVI siècle et ses personnages, Paris, Presses universitaires de France, 1978, p. 111.

26 Frédéric Deloffre et Michel Gilot, notice de le Chemin de la Fortune ou le Saut du fossé, dans Journaux et auvres diverses de Marivaux, Paris, Garnier, 1969, p. 329. 
relativement récente de la banqueroute du système financier de John Law et de ses conséquences pour les contemporains, dont Marivaux lui-même, qui a vu ses intérêts pécuniaires compromis durant cet épisode de la Régence. À ce titre, le Chemin de la Fortune ou le Saut du fossé peut certainement être rapproché d'une lettre des Lettres persanes de Montesquieu, qui a lui aussi mis en scène allégoriquement la faillite de John Law ${ }^{27}$.

Chez Marivaux, John Law est incarné par la figure de M. Rondelet. Résumons l'intrigue: le pauvre mais vertueux Lucidor et son complice La Verdure, vêtu d'une livrée étincelante, arrivent devant le palais de la déesse Fortune, auquel on ne peut accéder qu'en sautant un large fossé. Au bord du chemin gisent les tombes où le Scrupule a enterré les vertus de ceux qui ont franchi le fossé: «Voilà qui est plaisant, dit Lucidor à son valet, ci-gît la morale d'un philosophe et le désintéressement d'un druide. A ce que je vois, il y a ici une curieuse mortalité sur les vertus»; "Ah! c'est que les vertus ont la vie courte», rétorque La Verdure ${ }^{28}$. Après avoir décliné le chemin de l'honneur, un détour inutile selon La Verdure, les protagonistes se présentent à la Fortune qui les interroge pour savoir si elle doit les accepter dans son palais. D'emblée, la Fortune est séduite par l'étincelante livrée de La Verdure, et on doit voir là une allusion au paradigme machiavélien selon lequel la Fortune se livre de préférence aux gens virils, actifs, énergiques, courageux:

Interrogez-le avec bonté; je suis volontiers favorable aux mortels de son espèce; j'ai du faible pour eux. Je trouve celui-ci un joli garçon; il a je ne sais quoi d'ardent et de hardi dans la physionomie, qui me plaît. Son ajustement même est de mon goût; cet habit-là me gagne ${ }^{29}$.

Toutefois, la Fortune feint d'être contrariée par ces jeunes prétendants hésitant à laisser tomber leurs vertus, qui les appesantissent. Il en va tout autrement pour le prétendant suivant, M. Rondelet, alias John Law, grossier et cynique dans le récit de Marivaux et qui saute le fossé sans hésitation, car il n'est vraisemblablement encombré d'aucune vertu. La Fortune, «lui tendant les bras», lui dit: «Viens mon gros benêt; lourdaud,

27 Voir Montesquieu, Lettres persanes (1721), préface de Jean Starobinski, Paris, Gallimard, 1973, lettre CXLII, p. 319-324. Law est incarné par la figure d’Éole qui vend des outres pleines de vent en donnant l'illusion qu'il s'agit d'or.

28 Marivaux, le Chemin de la Fortune ou le saut du fossé, dans Théâtre complet, op. cit., p. 409.

29 Ibid., p. 411. 
mon ami, viens: je veux que tu ailles chez moi. Tu sauteras bien le fossé, toi; rien ne t'arrêtera: tu n'y entends point de finesse, et je te tiendrai la main moi-même. Saute; je vais aller te rejoindre ${ }^{30}$ ", avant de le laisser tomber, comme en témoigne la retentissante faillite de John Law.

D'Alembert, dans son Éloge de Marivaux, considère que ce qu'il a «surtout tracé d'une manière supérieure, [est] la fierté noble et courageuse de la vertu dans l'infortune, et le tableau consolant de la bienfaisance et de la bonté dans une âme pure et sensible ${ }^{31}$ », et il estime, en 1785, que les romans de cet auteur ont obtenu la préférence sur ses comédies. L'opinion du philosophe, sur ce dernier point, se distingue de l'opinion du public. Sous la Terreur, aux prises avec l'exaspération de passions où les ressorts de la séduction, de la manipulation et de la corruption menacent l'équilibre et l'existence même d'un corps politique, le public s'est fait complice du théâtre de Marivaux.

\section{MARTIN NADEAU}

École des hautes études en sciences sociales

30 Ibid., p. 412.

31 D'Alembert, Éloge de Marivaux, dans Théâtre complet, op. cit., p. 22. 